\title{
Correction to: Effects and Mechanism of Action of Panax notoginseng Saponins on the Pharmacokinetics of Warfarin
}

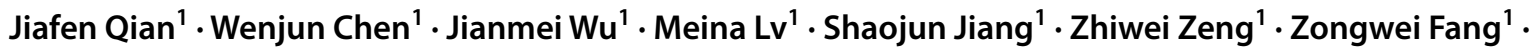 \\ Mingrong Chen ${ }^{1} \cdot$ Jinhua Zhang ${ }^{1} \mathbb{B}$
}

Published online: 22 February 2022

(c) Springer Nature Switzerland AG 2022

\section{Correction to: \\ European Journal of Drug Metabolism and \\ Pharmacokinetics \\ https://doi.org/10.1007/s13318-022-00753-0}

The original version of this article unfortunately contained a few typos in the abstract.

Abstract, First section heading, which previously read:

Backgroud

should read:

Background

Abstract, Results: 3rd sentence, which previously read:

Compared with the warfarin alone group, the clearance rate $(\mathrm{CL} / \mathrm{F})$ of warfarin in the warfarin + low-dose PNS, warfarin
+ medium-dose PNS, and warfarin + high-dose PNS groups was $10 \%(P>0.05), 23 \%(P<0.05)$, and 33\% $(P<0.05)$ lower, respectively, while the systemic exposure (area under the concentration-time curve, $\left.\mathrm{AUC}_{0-t}\right)$ increased by $106 \%(P$ $>0.05), 119 \%(P<0.05)$, and $134 \%(P<0.05)$, respectively, and the blood concentration of warfarin incresed by $112 \%$, $113 \%$, and $114 \%$, respectively (all $P>0.05$ ).

Should read:

Compared with the warfarin alone group, the clearance rate $(\mathrm{CL} / \mathrm{F})$ of warfarin in the warfarin + low-dose PNS, warfarin + medium-dose PNS, and warfarin + high-dose PNS groups was 10\% $(P>0.05), 23 \%(P<0.05)$, and 33\% $(P<0.05)$ lower, respectively, while the systemic exposure (area under the concentration-time curve, $\left.\mathrm{AUC}_{0-t}\right)$ increased by $106 \%(P$ $>0.05), 119 \%(P<0.05)$, and $134 \%(P<0.05)$, respectively, and the blood concentration of warfarin increased by $112 \%$, $113 \%$, and $114 \%$, respectively (all $P>0.05$ ).

The original article can be found online at https://doi.org/10.1007/ s13318-022-00753-0.

Jinhua Zhang

pollyzhang2006@126.com

1 Department of Pharmacy, Fujian Medical University Union Hospital, \#29 Xinquan Road, Fuzhou 350001, Fujian, China 